Estudios sobre armas antiguas, arte militar $\mathrm{y}$ vida cultural en oriente y occidente XXXI (2011), pp. 43-56

ISSN: 0436-029X

doi: 10.3989/gladius.2011.0002

\title{
LA CAMPAÑA EGIPCIO-CHIPRIOTA (383-373 A.C.): RELACIONES INTERNACIONALES Y MERCENARIOS GRIEGOS EN ORIENTE ${ }^{1}$
}

\author{
THE EGYPTIAN-CYPRIOT CAMPAIGN (383-373 B.C.): INTERNATIONAL RELATIONS \\ AND GREEK MERCENARIES IN THE EAST
}

POR

\author{
Daniel Gómez CAStro*
}

\section{RESUMEN - ABSTRACT}

Este artículo pretende llevar a cabo un análisis sobre el uso de mercenarios griegos en Oriente a partir de la Paz del Rey en 386 a.C. En un nuevo marco internacional refrendado por un pacto multilateral, Atenas utilizó a mercenarios con la finalidad de prestar apoyo, sin representar oficialmente a la ciudad, a enemigos de otras potencias en teoría aliadas como Esparta o Persia. El estudio concluye que la sofisticación de las relaciones internacionales junto al desarrollo de la técnica y estrategia bélica en el siglo IV a.C, potenció el uso de este tipo de soldado, convirtiéndolo así en una herramienta fundamental para cualquier estado con aspiraciones hegemónicas.

This paper wants to carry out an analytical description of the use of Greek mercenaries in the East, from King's Peace of 386 BC. In a new international world-system based on multilateral relations, Athens employed a shadow force of mercenaries in order to support the enemies of its allied powers, no matter if they were Sparta or Persia. Taking this example into mind, the radical improvements in war strategy along the IV century, as well as the high complexity of the international relations in the East, made such soldiers become an essential tool for any Ancient state with hegemonic aspirations.

\section{Palabras Clave - Keywords}

Relaciones internacionales; mercenariado; Paz del Rey; hegemonía; autonomía; Koiné Eirene.

International relations; mercenarism; Peace of King; hegemony; autonomy; Koiné Eirene.

En el año $392^{2}$, cuando Antálcidas fue incapaz de impulsar una paz con el gran rey Artajerjes II que permitiera a Esparta mantener su hegemonía en Grecia (X., $H G, \mathrm{IV}, 8,12-17)^{3}$,

1 El presente trabajo se ha realizado en el marco del proyecto HAR2010-19185, dirigido por el profesor Toni Ñaco del Hoyo. No quisiéramos dejar escapar la oportunidad de expresar nuestro agradecimiento a los profesores Toni Ñaco del Hoyo, Borja Antela Bernárdez, Jordi Cortadella Morral y, muy especialmente, a César Fornis Vaquero por las sugerencias y correcciones realizadas al artículo. Asimismo, también nos gustaría agradecer los comentarios realizados por profesor Fernando Quesada Sanz en el II Encuentro de Jóvenes Investigadores celebrado en la Sociedad Española de Estudios Clásicos (Madrid, 2008) y al evaluador anónimo de la revista por sus constructivas correcciones. Lógicamente, cualquier error en el texto debe ser únicamente atribuido a su autor.

* Universitat Autònoma de Barcelona $<$ danigomcas@gmail.com>.

2 Todas las fechas que se citen en el presente trabajo, con la excepción de las referencias bibliográficas de trabajos modernos y en los casos en que se especifique lo contrario, deben considerarse como antes de Cristo.

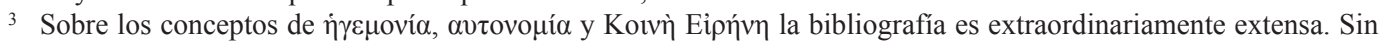
embargo, desde nuestra óptica resultan especialmente sugerentes los trabajos de Seager (1974: 36-63), Karavites (1984, 
todo apuntaba a que la pólis lacedemonia iba a perder la influencia conseguida tras su reciente victoria frente Atenas en la Guerra del Peloponeso. Sin embargo, cinco años más tarde, en 387, el Gran Rey y sus asesores entendieron que, frente a la peligrosa perspectiva de una segunda edición de la Liga de Delos que desestabilizara aun más el Mediterráneo Oriental, pactar con los lacedemonios, a quienes Artajerjes consideraba poco menos que «mentirosos blasfemos» (Dinon, FGrH $690 \mathrm{~F} 19)^{4}$, permitiría extender su influencia política sobre las póleis griegas ${ }^{5}$ y concentrar los recursos del Imperio en recuperar Egipto y la isla de Chipre (Briant, 1996: 666). Así pues, para obligar a Atenas a adherirse a la denominada Paz del Rey (o de Antálcidas) en 386, persas, espartanos y siracusanos bloquearon con su flota el paso marítimo del Helesponto, poniendo así en grave en peligro el abastecimiento de grano de Atenas, la cual no tuvo otro remedio que capitular oficialmente ese mismo año (X., $H G, \mathrm{~V}, 1,28)$.

En nuestra opinión, lo más interesante de los acontecimientos que acabamos de exponer es la urgencia, asumida por Artajerjes II, de llevar a cabo un proceso de relegitimación de su poder que sólo podía pasar por la victoria en el campo de batalla (Briant, 1996: 649; Plut., $A r t, 13,2)$. En este sentido, Artajerjes ya había visto cómo la revuelta de su hermano Ciro para arrebatarle el trono debilitó el Imperio hasta el punto de perder el control de Egipto y Chipre (Briant, 2006: 19). El soberano persa, en tanto que buen mazdeísta, entendía la mentira como una blasfemia y, por tanto, Evágoras de Salamina y Ácoris de Egipto eran mentirosos, pero además eran también rebeldes, lo cual, más allá de cualquier implicación religiosa, desgastaba políticamente la legitimidad de la soberanía persa, y la del mismo monarca, sobre el resto de pueblos que conformaban el Imperio (Briant, 2006: 18). Este proceso de relegitimación fundamentado en la victoria sobre quienes habían cuestionado la soberanía del Gran Rey fue la principal causa del inicio de una fase de expansión que culminó, por el lado griego, con la jura de la Paz del Rey ${ }^{6}$ y, por el persa, con la proyección de una potente campaña militar para la recuperación de los territorios escindidos de Egipto y Chipre ${ }^{7}$. Esto supuso el desarrollo de una forma de dominio basada en pactos que establecían un punto de partida político forzosa-

167-191), Moritani (1988: 573-77), Bostworth (1989: 122-151), Hansen (1995, 21-43), Antela (2007: 1-21) y, finalmente, Fornis (2007: 155-183; 2008: 298-327).

4 Entre los principales preceptos del zoroastrismo se encuentra el amor a la verdad y desprecio por la falsedad (Hdt, I, 136 y Olsmtead, 1948: 100), siendo el Gran Rey el mayor enemigo de la mentira (García Sánchez, 2005: 224; Briant, 1996: 259-260). Así pues, Artajerjes no sólo entendió como una traición la rotura por parte de Esparta de los múltiples pactos entre lacedemonios y aqueménidas (el más importante de los cuales fue el de Beocio en 409), sino que, al defender a las ciudades que habían respaldado militarmente a Ciro (cuya estrategia se había fundamentado en el engaño, vid. X., $A n$., I, 1, 6-8), se postulaba como un enemigo de la verdad que trataba de evitar la purificación de un acto impío como era la falsedad y, como veremos más abajo, un intento por desgastar la legitimidad de su poder.

5 El nuevo tratado (X., $H G, \mathrm{~V}, 1,31$ ) suponía el reconocimiento de Persia como mediadora en los conflictos entre las potencias griegas y, a la vez, debilitaba la articulación interna de la Hélade en favor de la hegemonía espartana (Karavites, 1984: 181). La ruse que permitía mantener la mencionada hegemonía lacedemonia era la cláusula de la autonomía de las póleis griegas, pues no en vano se cumplieron los temores del resto de potencias helenas al quedar explícitamente prohibida toda asociación de ciudades o ligas a excepción de la Liga del Peloponeso (X., $H G, I V, 8.15$ ). Hay que tener en cuenta que, en este período concreto, el significado del concepto «autonomía»se contrapone a eleutheria, pues el primero únicamente expresaría una forma de dominio imperialista al ser ésta simplemente una concesión instrumentalizada por parte del poderoso (Plácido Suárez, 2001-2002: 202; Hansen, 1995: 25). En este aspecto, la diplomacia, moderna y antigua, es el arte de decir lo que los demás esperan escuchar (Schmidt, 1999: 86), pero, en realidad, los espartanos querían para las ciudades de Asia Menor la misma autonomía que tenían las ciudades del Peloponeso, es decir, la que ellos consideraban oportuna (Orsi, 2004: 57, n. 60; X., HG, III, 2, 30-31). En cuanto al significado concreto de autonomía que se impuso en este tratado, vid. Cawkwell, 1981: 72. Respecto al debate general del concepto, así como sus diferentes significados y las implicaciones políticas del mismo, resulta fundamental el trabajo de Hansen (1995: 21-43).

6 Artajerjes, al asegurarse, gracias al tratado de paz, de que ninguna potencia griega le disputaría la soberanía sobre Asia Menor, dio por cerrada su «política griega» y, de este modo, pudo centrar sus esfuerzos en recuperar Chipre y Egipto (Fornis, 2007: 166-167).

7 Evágoras de Salamina de Chipre no había roto oficialmente su vasallaje para con el Gran Rey. Sin embargo, la conquista por parte del monarca heleno de toda la isla podía perjudicar la campaña en Egipto y fue el propio Artajerjes 
mente aceptado por todas las potencias en liza ${ }^{8}$. En este escenario, la única posibilidad que les quedaba a Estados con aspiraciones hegemónicas como Atenas para mantenerse dentro de la legalidad era recurrir a mercenarios que llevaran a cabo las acciones necesarias para alcanzar sus objetivos políticos reales. Esto explicaría el auge de este tipo de servicio militar tras la Guerra del Peloponeso, más allá de las razones tradicionalmente aducidas que han visto en el mercenariado únicamente un residuo de las diferentes crisis socio-económicas acaecidas en Grecia tras el conflicto entre lacedemonios y atenienses ${ }^{9}$. En este sentido, la campaña egipciochipriota entre los años 385-373 deviene un caso paradigmático de la nueva situación internacional creada con la jura de la Paz del Rey ${ }^{10}$.

\section{ORÍGENES DE LA REVUELTA}

La revuelta de Ciro (401) otorgó a los gobernantes antipersas de Egipto una nueva oportunidad para intentar sacudirse definitivamente el yugo aqueménida, tratando así de finalizar el proceso iniciado por Amirteo, quien, aprovechando la muerte del rey Darío II en 404 promovió una revuelta que desplazó del poder a la etno-clase filopersa dominante (Briant, 1988: $137)^{11}$. No es este el lugar para enumerar las importantes lagunas que presenta el estudio del Egipto aqueménida ${ }^{12}$, sin embargo, resulta imprescindible realizar una breve explicación de

quien declaró la guerra a Evágoras (Isoc., IX, 67-68; D.S., XIV, 8), pues cualquier campaña dirigida contra el país del Nilo sin el control efectivo de Chipre hubiese significado un fracaso de antemano (Shrimpton, 1991: 5; Briant, 1996: 666).

8 Se considera como el primer pacto multilateral en Grecia (Ryder, 1965; Schmidt, 1999: 82) así como el origen de todos los tratados de paz que se llevarán a cabo con posterioridad bajo el instrumentalizado concepto político de Koinè Eiréne (Schmidt, 1999: 83-85). Sin embargo, como bien ha señalado algún autor, al ser necesaria la coerción para que potencias como Atenas respetaran el tratado, éste no puede entenderse como un «pacto multilateral» propiamente dicho, sino más bien como un ultimátum (Fornis, 2007: 157), idea que respaldarían las fuentes al catalogar el pacto como «la paz enviada por el Rey» (X., HG, V, 1, 30, 35; Dem., XV, 9, 29, XX, 54; Plb., I, 6, 2).

9 Ha sido común concebir al mercenario griego del siglo IV como una consecuencia de la situación de pobreza general en que se encuentra Grecia tras los casi 30 años de duración de la Guerra del Peloponeso. En este sentido, resulta célebre la expresión de «patología social» para referirse a este tipo de soldado (Garlan, 1972: 95). Esta explicación, sin embargo, aborda un fenómeno multidimensional como éste desde un único aspecto y, por tanto, en nuestra opinión, sólo lo resuelve parcialmente (Trundle, 2004: 63). Puede encontrarse un análisis teórico y una revisión de la concepción tradicional de mercenariado en Gómez (2010: 95-116).

10 Cabe señalar que, si bien la estrategia basada en el uso de mercenarios por parte de Atenas en la Campaña EgipcioChipriota fue el primer conflicto internacional en que se utilizaron mercenarios de forma encubierta tras la firma de la Paz del Rey, esta estrategia ya había sido usada por Esparta con anterioridad. Ciro el Joven había ayudado enormemente a los lacedemonios en la última fase de la Guerra del Peloponeso (X., $H G, \mathrm{I}, 5,1-7 ; 6,18$; II, 1, 7-15; 3, 8) y, por ello, cuando el joven príncipe exigió a Esparta que le devolviera la ayuda que él les había prestado para deponer a su hermano Artajerjes II (X., $H G$, III, 1), ésta se vio en la obligación de ayudarle tratando de evitar en todo lo posible enemistarse con el Gran Rey, aliado suyo en aquellos momentos. Es decir, la solución pasó por apoyar el envío de un contingente de mercenarios dirigidos por un espartiata, Clearco, quien, a pesar de ser un exiliado, estaba vinculado a la facción política del desaparecido rey Agis, (X., $H G, \mathrm{I}, 1,36 ; 3,15)$, más tarde integrada en la facción de Agesilao, hermano de Agis, y Lisandro, amante y mentor de Agesilao (Plut., Ages., II, 1). La efectividad de las tropas griegas hizo evidente la utilidad del uso de mercenarios para todas las potencias, es decir, de tropas bien entrenadas que, sobre todo, podían ser reclutadas rápidamente (Trundle, 2004: 72 y ss).

11 La principal fuente que nos habla de Amirteo y sus sucesores es la Crónica Demótica, texto papiráceo del siglo II que actualmente se encuentra en la Biblioteca Nacional de París (Pap. Dem. n. ${ }^{\circ}$ 215). Es consultable en traducción al inglés en $<$ http://www.yorku.ca/pswarmey/2110/dem-chron> (Universidad de York). El sucesor de Amirteo, Neferites I (398-392), primer Faraón de la dinastía XXIX, se alió inmediatamente con Esparta para tratar de mantenerse independiente de la soberanía aqueménida. Tras su muerte, se produjo en Egipto una fuerte lucha por el poder seguida por numerosas revueltas campesinas, lo cual denotaría la ausencia de legitimidad política que dicha dinastía tenía ante la población egipcia (Briant, 1988: 157).

12 Puede encontrarse un perfecto planteamiento de la cuestión en Briant, 1988, pp. 137-173 y, más recientemente, en la interesante revisión del caso realizada por el mismo autor en 2003, pp. 33-47. 
sus principales dificultades. Entre éstas destacaremos dos: el argumento de la «revuelta nacional egipcia» (Mallet, 1922: 33) y la ausencia de rigor científico en el análisis del registro arqueológico ${ }^{13}$. Estas dos cuestiones son fundamentales porque propiciaron una corriente de pensamiento general entre los egiptólogos según la cual se consideraba el período de dominación persa como una anomalía que habría provocado un estatismo socioeconómico y cultural en el país del Nilo que sólo pudo superarse tras la expulsión de los aqueménidas y la recuperación de su natural desarrollo. Respecto a la cuestión de la «revuelta nacional», cabe señalar que, como veremos más detalladamente, los campesinos se rebelaron, más que contra un invasor extranjero, contra una presión fiscal insoportable, razón por la cual las revueltas en Egipto se fueron sucediendo hasta época helenística (Briant, 1988: 142). Si todo se centra en el argumento de la «revuelta nacional», se soslayan cuestiones fundamentales de la situación histórica como, por ejemplo, contra quiénes iban dirigidos los levantamientos armados; y se falsean aspectos claves como la interrelación entre Estados y las políticas socioeconómicas de una potencia imperial sobre un territorio que, además de una personalidad cultural propia, tenía sobre todo una jerarquía social fuertemente asentada y, por tanto, una clase dirigente ancestral que desde siempre se había beneficiado del ejercicio del control de los recursos económicos del Estado. Como decíamos, entendemos que el aumento de la fuerte explotación tributaria ${ }^{14}$, que provocó miseria y hambre, fue la causa real de las revueltas, y ésta era llevada a cabo a nivel local por las elites egipcias colaboracionistas (los «dinastas») más que por la Administración persa propiamente dicha, pues parece demostrado que los templos y los grandes propietarios continuaron detentando suficiente poder para imponer sus propios tributos ${ }^{15}$. A pesar de esto, cabe señalar que en Egipto existían importantes diferencias regionales y las sublevaciones no respondieron siempre a las mismas causas. Por ejemplo, el conflicto judeoegipcio de la ciudad nilótica de Elefantina tenía poco en común con las revueltas acaecidas en el Delta (Briant, 1988: 144).

Por consiguiente, resulta necesario establecer qué regiones y, dentro de las mismas, qué estamentos sociales tomaron parte en las revueltas. Como es sabido, es precisamente de la zona deltaica de donde parten todas las revueltas dirigidas por una aristocracia de carácter antipersa y, por tanto, realmente organizadas tanto a nivel político como militar ${ }^{16}$. Debido al difícil control de esta área, los persas se vieron obligados a utilizar dinastas locales para ejercer un control más efectivo del territorio (Hdt., III, 15) ${ }^{17}$. Una de las principales dificultades que

13 Dicha «ausencia de rigor científico» en el análisis del registro arqueológico partiría de la idea general de que la dominación persa de Egipto fue simplemente una anomalía sin ningún tipo de influencia real sobre la cultura egipcia (Briant, 2003: 39). Así, por ejemplo, se ha considerado que el cambio en las costumbres funerarias egipcias por parte de un grupo determinado a partir del 525 se debe a un cambio súbito sin ninguna influencia persa. Por esta razón, existen autores que denuncian la mencionada «ausencia de rigor científico en el análisis del registro arqueológico» al considerar que muchos de los materiales (sobre todo cerámicas) atribuidos a la época saíta serían de época de dominación persa (Aston, 1999: 17).

14 Hecho del que deja constancia Diodoro Sículo (XI, 71, 3), aunque éste acuse directamente a la Administración persa (también en XVII, 49, 1).

15 Esta situación contribuyó a la creación en Egipto de una contradicción interna entre dinastas filopersas y dinastas antipersas (Briant, 1988: 140 y 155).

16 Como pone de manifiesto Briant (1988: 148), esto no se debe a una mayor «consciencia nacional» de la zona pues, al final, las dinastías egipcias establecidas eran de origen libio, sino a que se trataba de una región de difícil control para los poderes centrales. La tesis del autor francés se centra en la importancia del Delta en las relaciones internacionales del Mediterráneo Oriental, ya que con la fundación de la Liga de Delos en 470, éste deviene una zona importante para definir la fuerza o la debilidad económica de los aqueménidas en esa región, razón por la cual los atenienses hicieron todo lo posible por promover insurrecciones. Las revueltas serían, pues, de carácter exógeno, siendo un ejemplo de esto la revuelta de Inaros (460) narrada por Tucídides (I, 104-109, 3). Inaros sabía que sin la ayuda ateniense no podía hacer frente al Gran Rey y, por eso, según Diodoro (XI, 71, 4), éste prometió el control de una parte del territorio egipcio a Atenas.

17 Esto demostraría la incapacidad persa de controlar esta vital zona de Egipto e, incluso, retóricamente, se podría preguntar si los persas realmente «conquistaron» el territorio (Briant, 1988: 150 y 172). 
dichos dinastas causaban a los persas era que poseían una «política exterior» propia que, a menudo, iba en contra de los intereses aqueménidas ${ }^{18}$. Además, tras la expulsión de los persas, las guerras y la contratación de mercenarios debieron provocar un aumento mayor de la presión fiscal. Seguramente por esta razón se dieron las condiciones para un importante aumento de la conflictividad social y política que provocó numerosos asaltos al poder ${ }^{19}$. En cuanto a la comúnmente aceptada «persofobia» egipcia, es necesario señalar que ésta fue fruto de la ideología de los historiadores de época helenística, los cuales tenían la intención de mostrar al mundo a un Alejandro «liberador» del yugo aqueménida (Briant, 1988: 152) ${ }^{20}$.

El caso de Chipre es menos complejo ya que la fractura se produjo por el lado persa a causa de razones geoestratégicas relacionadas con la campaña de recuperación de Egipto. A finales del siglo V Chipre estaba dividida al menos en dos áreas de influencia, una de tendencia fenicia y otra filoateniense ${ }^{21}$. Salamina de Chipre era una pólis helena cuyo rey, Evágoras, había accedido de nuevo al poder en 410 tras unos años en el exilio (D.S., XIV, 8). Después de recuperar su posición, Evágoras puso en marcha un plan para tratar de alcanzar rápidamente sus ambiciones políticas, a saber, extender su soberanía a todas las ciudades de la isla (Briant, 1996: 666). Así pues, para eliminar toda influencia espartana de Chipre, se alió con el Gran Rey en 398 e incluso contribuyó con naves a la victoria persa de Cnido en 394. La Batalla de Cnido, precisamente, supuso un punto de inflexión en sus relaciones con Artajerjes al producirse un reajuste en la estrategia persa en el Mediterráneo Oriental, la cual no contemplaba una Chipre unificada bajo la soberanía de Evágoras. Para el rey heleno de Salamina de Chipre, el principal problema era que Artajerjes, tras lo ocurrido en Cunaxa (401), se preocupó especialmente por evitar la formación de cualquier poder que desestabilizase Asia Menor y, sobre todo, que pudiese dificultar las acciones marítimas de la flota durante las futuras campañas para recuperar Egipto (Briant, 1996: 667; Shrimpton, 1991). Chipre, a nivel geoestratégico, se habría convertido durante el siglo $\mathrm{V}$ en la pieza clave para controlar de forma efectiva Asia Menor, Levante y Egipto. Haciendo la lectura inversa, podemos decir que para controlar de forma efectiva Chipre era necesario dominar todo el Mediterráneo Oriental (Petit, 1991: 169). Tras la formación de la Liga de Delos en 477, los ataques contra Chipre fueron cíclicos ${ }^{22}$. Junto con Egipto, la isla se convirtió en el eje central de la política internacional en su área geográfica, sobre todo en relación con la situación estratégica de Egipto, cuya suerte estuvo irremediablemente ligada a la potencia que controlaba de forma efectiva la isla mediterránea ${ }^{23}$. De este modo, Chipre, convertida en una potencia dirigida por un monarca filoateniense como

18 Prueba de ello es el cargamento de grano que Psamético envió a Atenas en 445 (Th., I, 104-109), lo cual también evidenciaría su dominio sobre las zonas fértiles de Delta. Así pues, como sugeríamos más arriba, esta debilidad política persa habría aumentado durante la crisis provocada por Ciro el Joven y, al final, ésta pudiera haber sido la causa de la degradación y hundimiento del dominio aqueménida en Egipto entre el 404 y el 399 (Briant, 1988: 150).

19 A la muerte de Neferites en 392, se abrió una fuerte pugna entre aristócratas por la sucesión del reino (Mutis y Psamutis). Ambos se autoproclamaron faraones a la vez, lo que fue aprovechado por Ácoris (un pariente de Neferites) para sublevarse, derrotar a las otras dos facciones y establecerse finalmente como faraón en 391.

20 La Crónica Demótica, por ejemplo, ataca por igual a persas, templos y faraones (Dinastías XXIX y XXX) por ejercer una presión fiscal que dejó a los campesinos en la miseria.

21 Como ocurría en el resto de territorios dominados por los aqueménidas, los altos cargos, y nos referimos en este caso al sátrapa de la zona, eran miembros de la alta aristocracia persa, pero la administración de las ciudades corría a cargo de las dinastías locales de la isla (Petit, 1991: 163).

22 Además del ataque en 478, Tucídides (I, 104, 1-2; 112, 1-4) nos relata dos razias más.

23 Diodoro (XII, 4,1 ) ya habla de una «importante guarnición persa» con motivo de la expedición dirigida por Cimón en 450. Sin embargo, esta información ha sido puesta en cuestión por parte de la historiografía moderna, pues Evágoras consiguió hacerse en 390 con el control de la isla fácilmente sin ningún tipo de oposición persa (Petit, 1982: 165, n. 14). El propio Diodoro (XI, 44, 2) cita algunas con motivo de la campaña helena liderada por el «regente» espartano Pausanias en 477. Sin embargo Tucídides, que ubica la misma expedición en 478, no nombra ninguna. 
Evágoras, podía no sólo complicar la campaña de recuperación de Egipto, sino desestabilizar toda Asia Menor ${ }^{24}$.

Así pues, el Gran Rey decidió poner freno al riesgo que suponían las ambiciones de Evágoras una vez neutralizado el peligro heleno. En primer lugar, emprendió la guerra contra Esparta para desarticular el incipiente imperio marítimo espartano ideado por Lisandro tras la batalla de Cnido en 394. En segundo lugar, frenó durante unos pocos años las ambiciones atenienses de editar una segunda liga marítima con el tratado de paz del 386. Como decíamos, una vez desarticulado el frente griego, Artajerjes esgrimió como casus belli la subyugación de sus súbditos en las ciudades fenicias de la isla (D.S., XIV, 8) y, de esta forma, el rey de Salamina de Chipre no tuvo más opción que aliarse con los enemigos del Gran Rey (fundamentalmente Ácoris de Egipto) para tratar de frenar la maquinaria de guerra aqueménida (D.S. XV, 29, 1).

\section{LA CAMPAÑA EGIPCIO-CHIPRIOTA Y LOS CONTINGENTES MERCENARIOS EN LOS EJÉRCITOS}

El Gran Rey declaró la guerra a Evágoras en el $390^{25}$, pero la guerra contra Esparta provocó que la campaña contra Chipre no se iniciara hasta que no se solucionaron los asuntos griegos con la Paz del Rey en 386. Las potencias griegas reconocían a través del tratado de paz la legitimidad persa de controlar Chipre y así, oficialmente, Esparta y Atenas condenaban a Evágoras, el «campeón del helenismo» en la isla, a permanecer bajo soberanía aqueménida (Isoc., IX, 47). De esta forma, el ateniense Cabrias, quien se encontraba en Salamina de Chipre con unos ochocientos peltastas mercenarios, diez trirremes y un número sin determinar de hoplitas desde $388(\mathrm{X}, H G, \mathrm{~V}, 1,10)^{26}$, se vio obligado a salir de la isla y optó por unirse al rebelde Ácoris en Egipto. La invasión de Chipre se produjo en el mismo 386 . El primer enfrentamiento, conocido como Batalla de Citium, demostró la superioridad bélica persa y, lógicamente, Evágoras fue derrotado (D.S., XV, 3). Tras la victoria, Tiribazo, almirante de la flota persa, y Orontes, el sátrapa de Armenia, comandante del ejército terrestre, pudieron invadir completamente la isla y sitiar Salamina de Chipre ${ }^{27}$. Evágoras huyó ese mismo año a Egipto y allí intentó conseguir soldados y oro para hacer frente al sitio de su ciudad, pero no consiguió el apoyo pleno de Ácoris, quien le ayudó de forma simbólica con una modesta cantidad de oro (D.S., XV, 4, 3; 8, 1).

24 En aquel momento tanto Trasíbulo como Conón, habían tratado de reconstruir el imperio marítimo ateniense (Cawkwell, 1976: 270), lo que supuso una de las causas por las cuales los persas cambiaron su política de alianzas; arrestaron a Conón (X., $H G$, IV, 8, 16; D.S, XIV, 85, 4) y obligaron a los atenienses a firmar la Paz del Rey.

25 Existe un grave problema de datación en la guerra contra Evágoras debido a la información contradictoria de las fuentes. Por un lado, Diodoro (XV, 9, 2) informa que la campaña duró diez años, pero que la mayor parte del tiempo se utilizó en gestionar los preparativos, de modo que la invasión del 386/5 sólo duró dos años (aunque Evágoras se rindió en 381 según los «Informes astronómicos babilónicos»). Isócrates, por su parte, mientras nos dice que el conflicto duró diez años en su Evágoras (IX, 64), en su Panegírico (IV, 135-141) afirma que el Gran Rey llevaba seis años sitiando Salamina de Chipre sin éxito. Es precisamente aquí donde aparecen los problemas, pues parecería lógico ubicar este último discurso en 380 , pero ha sido fechado en 384. Según la explicación tradicional, Isócrates fue actualizando su discurso durante el tiempo que duró la campaña egipcio-chipriota, de tal forma que sólo estuvo completamente acabado en 380. Una exposición detallada del problema así como de las diferentes posturas historiográficas puede encontrarse en Stylianou, 1998: 143 y ss. En cuanto a la cronología, nosotros utilizamos en la medida de lo posible los «Informes astronómicos babilónicos», ya que consideramos que resultan una fuente más fiable al ser contemporánea a los hechos y no tener ningún tipo de finalidad política (Van der Speck, 1998).

26 Trundle $(2004,48)$ entiende que los tres mil peltastas de que disponía Evágoras para defender su ciudad eran mercenarios (Isoc., IV, 141), pero no existe ningún otro dato que lo corrobore.

27 Es importante destacar que Artajerjes lideró las tropas in situ (D.S, XV, 2, 1), lo cual ha sido respaldado por la documentación existente y las tablillas de los «Informes astronómicos babilónicos» (Briant, 1996: 1010-1011). 
En 384, tras dos años de lucha, la situación era desesperada para el rey de Salamina de Chipre; por un lado había perdido el control de la isla y su ciudad estaba siendo sitiada (D.S., $\mathrm{XV}, 3,4-6)$ y, por otro, ya no podía esperar más ayuda por parte de sus aliados. Por esta razón empezó a negociar con Tiribazo las condiciones para una hipotética rendición (D.S., XV, 8, 2). Sin embargo, las duras imposiciones de Tiribazo provocaron las quejas del rey chipriota y del mismo Orontes ante el Gran Rey, por lo que éste destituyó del mando a Tiribazo y ordenó su arresto (D.S., XV, 8, 3-9). Con Tiribazo fuera de escena, los mandatarios persas consiguieron un tratado de paz en 381 que devolvía la situación de la isla al estado anterior a las operaciones militares de Evágoras; así Artajerjes lograba su principal objetivo, a saber, el cierre del frente de Chipre para iniciar el asalto a Egipto (Briant, 1996: 671) ${ }^{28}$. Sin embargo, esta resolución del conflicto en Chipre provocaba la humillación pública de un hombre fuerte como Tiribazo, lo cual generó todavía más problemas para el Persa, pues Glos, el hijo político legalmente reconocido por Tiribazo, se sublevó en 384 tras conocer la suerte del sátrapa, llevándose consigo una parte de la flota destinada a la campaña egipcia y privando de apoyo marítimo a Farnabazo, el encargado de las operaciones terrestres en Egipto, lo que a la postre haría fracasar el primer intento de recuperación (D.S., XV, 9, 3-5) ${ }^{29}$. En 380 todo favorecía las intenciones del Gran Rey de acabar con las aspiraciones de los rebeldes egipcios ${ }^{30}$, pero, de nuevo, la situación empezó a complicarse en Grecia y Artajerjes, en calidad de juez de los asuntos griegos a raíz de la Paz de Antálcidas, se vio obligado a intervenir, retrasando así la campaña egipcia. $\mathrm{El}$ asunto urgente que reclamó la atención persa fue que la tiranía filo-espartana instaurada en Tebas (X., $H G, \mathrm{~V}, 41)$ cayó en 379, lo cual propició la instauración de un régimen democrático en la ciudad beocia. Estos demócratas, por otro lado, habían surgido de la facción oligárquica antilaconia durante su exilio en Atenas (Pascual González, 1986: 77 y) que, dado el marcado desprestigio en la ciudad del sector oligárquico filolaconio (Pascual González, 1986: 76), podía desestabilizarse gravemente en contra de los intereses espartanos el equilibrio de fuerzas en Grecia.

La incapacidad espartana para someter a Tebas favoreció especialmente a Atenas, que inició conversaciones para reanudar las relaciones con los antiguos aliados, respetando formalmente los términos de la Paz del Rey. El método ateniense consistió en llegar a acuerdos bilaterales con Quíos, Rodas, Bizancio y Tebas para evitar así que este tipo de alianza pudiera parecer una confederación o liga, tipo de agrupaciones expresamente prohibidas por los tratados de paz firmados con el Persa. Además, se reconoció la autonomía de los aliados en los términos en que se contemplaba en la paz, es decir, la imposibilidad de cobrar tributos (aunque se mantenía la trampa legal de aceptar «contribuciones voluntarias»), ni destacar guarniciones militares en sus territorios. Mientras Esparta trataba en vano de reducir a Tebas, la Calcis de Eubea y algunas ciudades tracias se sumaron a Atenas, lo cual forzó a la pólis lacedemonia a actuar con su flota por el Egeo. Sin embargo, ésta fue completamente destruida por la ateniense el 376 frente a las costas de Naxos. La victoria ateniense provocó que en 375 Corcira, Cefalonia, Acarnania y otros pequeños estados del mar Jónico se sumaran a la alianza para

28 Los persas controlaron tan férreamente Chipre que ni siquiera existió rebelión alguna durante la conocida como Gran Revuelta de los sátrapas; posterior al primer intento serio de recuperación de Egipto por parte de Artajerjes II (D.S., XV, 90, 2-4).

29 Farnabazo realizó una infructuosa campaña contra Egipto entre 386-384, pero tuvo que retirarse al perder su apoyo marítimo (Isoc., IV, 140). Glos proyectó una alianza con Ácoris y los espartanos, pero fue asesinado antes de llevar a cabo sus planes.

30 Así lo indicarían las presiones que sobre Atenas hicieron los representantes persas para obligar a Cabrias, que se encontraba en Egipto, a regresar a Atenas el mismo año 380 y a enviar un contingente de mercenarios a las órdenes de Ifícrates como apoyo a las tropas del Gran Rey (D.S., XV, 29, 4), aprovechando la situación creada tras las diferentes crisis internas que se sucedieron en Egipto a la muerte de Ácoris a finales del 380. 
crear un nuevo poder marítimo en el Mediterráneo Oriental. Artajerjes no podía consentir esta nueva situación y proyectó una reunión de Estados griegos para asegurar la paz, disolviendo todo tipo de hegemonía griega en la Hélade ${ }^{31}$. Aun así, en 374, cuando Esparta, en un intento de frenar a Tebas, reconoció la legitimidad de la existencia de una «libre asociación de Estados» con Atenas a la cabeza, parecía que el nuevo equilibrio de fuerzas calmaría lo suficiente la situación para que Persia pudiese iniciar la deseada campaña en Egipto ${ }^{32}$.

Finalmente, el Gran Rey atacó Egipto en 373 con el cuerpo de mercenarios griegos más grande jamás contratado por un rey persa ${ }^{33}$. Nectanebo, el sucesor de Ácoris, había dotado de potentes defensas el Delta y sus principales vías de acceso (D.S., XV, 42, 1). No obstante, Farnabazo logró remontar el Nilo y desembarcó sus tropas en su orilla oriental, causando numerosas bajas entre la población civil y saqueando algunos templos. Ifícrates, comandante de los mercenarios griegos, propuso al sátrapa avanzar lo más rápidamente posible hacia Menfis para evitar el levantamiento de nuevas defensas, pero Farnabazo prefirió asentarse en la ciudad nilótica de Mendes y convocar a sus comandantes en asamblea para decidir la estrategia a seguir ${ }^{34}$. Sin embargo, a finales del 372, el Nilo creció de forma tan intensa que obligó a abandonar el campamento, razón por la cual Ifícrates, viendo la posición de debilidad en que habían quedado sus tropas, decidió huir con sus mercenarios durante la noche (D.S., XV, 43, $4)^{35}$. En esta coyuntura, Farnabazo ordenó la retirada del resto de las tropas al centro general de operaciones instalado en Palestina, consumándose un nuevo fracaso persa en Egipto.

Parece importante destacar que la idea tradicional de una derrota total persa durante la invasión egipcia no es exacta. Analizando la cuestión de forma general, la campaña alcanzó gran parte de los objetivos programados por los persas y, por tanto, debe considerarse como exitosa. Por un lado, se había recuperado Chipre y el levante Mediterráneo (en especial Tiro, que se había unido a Evágoras) y, por otro, se había conseguido la obediencia de las ciudades griegas en Europa $^{36}$. Si bien la conquista de Egipto se podía reemprender en cualquier momento desde Palestina, poner en orden la situación en que se encontraban las ciudades griegas se convirtió en el principal objetivo del Gran Rey. En este sentido, aliándose con Esparta, Persia centraba todos sus esfuerzos políticos en mantener la desunión griega en el continente. Los problemas

31 Esta asamblea de pólis terminó celebrándose en 371 y sirvió para enfrentar todavía más a las partes contendientes: Tebas se presentó en representación de toda Beocia, cosa que Atenas y, sobre todo, Esparta consideraron inaceptable. Como solía ocurrir, las diferentes posturas terminaron resolviéndose en el campo de batalla, dando como resultado Leuctra.

32 Diodoro $(\mathrm{XV}, 38,1)$ opina que el Gran Rey se vio obligado a proyectar una paz entre las ciudades helenas para poder acceder a la contratación de un elevado número de mercenarios griegos para su campaña egipcia. En nuestra opinión, si bien esto puede ser verosímil hasta cierto punto, es difícil pensar que esta situación pudiese darse en la Persia de principios del IV y, por tanto, parece que Diodoro estaba pensando más en la situación creada tras lo que él mismo definió (XV, 93, 1) como «Gran Revuelta de los sátrapas», donde las urgencias provocadas por el estado de crisis general en el imperio pudo favorecer este tipo de contrataciones masivas.

33 Diodoro (IV, 29, 4) cifra el ejército persa en 200000 hombres (de los cuales 20000 eran mercenarios griegos comandados por Ifícrates) y 300 naves. Nepos (XI, 2, 4) cifra el número de mercenarios de Ifícrates en 12000.

34 Para Diodoro $(\mathrm{XV}, 41,2)$ el largo tiempo invertido en los preparativos de la guerra fue lo que hizo fracasar la expedición, aunque para Briant (1996: 673) esta explicación sólo reflejaría la idea griega de la incapacidad bélica persa (respecto a la negativa del sátrapa de seguir la estrategia de Ifícrates vid. D.S., XV, 43). En cuanto a la presentación de los hechos que hace Diodoro, cabe señalar que sigue la idea general establecida por algunas fuentes del siglo IV, a saber, que los persas eran incapaces de ganar una batalla sin el asesoramiento griego (X., Cyr., VIII, 26).

35 Coincidimos con Briant (1996: 674) en que parece inverosímil que Farnabazo, ni ninguno de sus generales, no tuviera en cuenta el período de crecimiento del Nilo, lo cual plantea dudas sobre la fiabilidad histórica del episodio.

36 A pesar de la colaboración a título personal de Cabrias con Evágoras y Ácoris/Nectanebo, el mercenario ateniense había sido retirado por la ciudad tras las protestas persas. Además, Atenas colaboró con soldados en la invasión de Egipto e, incluso, Farnabazo, a pesar de su derrota, se sintió suficientemente fuerte para pedir a los atenienses el envío de Ifícrates para ser juzgado por su huida, petición que fue desestimada «muy amablemente» por la pólis ática (D.S., XV, 43, 6). De esta forma Artajerjes II devino nuevamente árbitro formalmente reconocido de los asuntos griegos. 
se multiplicaron para el Gran Rey en 372, cuando cesó a Farnabazo y puso en su lugar a Datames como el general encargado de la recuperación de Egipto. Éste aprovechó la comandancia del ejército para sublevarse contra Artajerjes y, pactando con los sátrapas de las regiones más occidentales, organizó una rebelión en cadena. Aunque a la postre los pactos entre sátrapas rebeldes resultaran frágiles e inestables, supusieron para el Gran Rey un nuevo frente mucho más acuciante que la recuperación de Egipto.

\section{CONTINGENTES MERCENARIOS}

Como se ha visto, existieron contingentes mercenarios en los dos bandos involucrados en la disputa y durante diferentes momentos y, por tanto, consideramos oportuno realizar un comentario pormenorizado de la información que nos ofrecen las fuentes. El primer episodio importante es el envío por parte de Atenas de Cabrias con un contingente de mercenarios formado por ochocientos peltastas y diez trirremes para ayudar a Evágoras en 388 (X., $H G, \mathrm{~V}$, $1,10)^{37}$. Éstos se unieron a los tres mil peltastas que el rey de Salamina de Chipre tenía para defender la ciudad (Isoc., IV, 141). Estos peltastas han sido considerados por algunos autores como mercenarios (Trundle, 2004: 48), pero Isócrates no lo especifica. Aun así, es posible que algún contingente mercenario no ateniense entrenado por Cabrias se quedara en la ciudad, pero, como decíamos, no existen pruebas que lo certifiquen y, en última instancia, también podían ser ciudadanos de la pólis chipriota entrenados como peltastas por el general ateniense. En cualquier caso, tras su salida de la isla con motivo de la Paz del Rey, Cabrias fue contratado por Ácoris para defender Egipto ${ }^{38}$. Sabemos que Cabrias trabajó en las fortificaciones del Delta en Egipto desde 386 hasta su retirada en 380/79 sin llegar a entrar en combate directo con las tropas persas (Stylianou, 1998: 150). Cuando Atenas retiró la ayuda a Ácoris, los atenienses enviaron a Ifícrates para participar en la guerra junto al Gran Rey contra los rebeldes egipcios, con veinte mil mercenarios (D.S., XV, 38, 1). Su principal papel dentro del ejército persa era llevar a cabo el entrenamiento de los mercenarios (Nepos, Ificrates, 2, 4), sobre todo de los que habían sido recientemente incorporados. Sabemos por Diodoro $(X V, 43)$ que Ifícrates también pudo efectuar una labor consultiva para Farnabazo, el comandante supremo del ejército persa, pero su opinión no tenía más peso que la de los demás generales medos. Además, a juzgar por la petición de castigo que presentó el sátrapa a Atenas por la huída de Ifícrates, parece que el estratego ateniense no gozaba de ningún tipo de autonomía dentro del ejército (D.S., XV, 43, 6).

Un último aspecto a destacar de este episodio bélico es la consideración del mismo como el primer caso importante de contratación de mercenarios griegos por potencias del Mediterráneo Oriental (Tourraix, 1999: 210; Cartledge, 1987: 323). Si nos fijamos en los datos exactos que las fuentes nos reportan, Cabrias había sido enviado a Chipre con ochocientos peltastas y diez trirremes en 388; mientras que Ifícrates lo hizo con veinte mil mercenarios en 386 . Si a

37 Sin embargo, antes de Cabrias, Filócrates había sido enviado por la ciudad para ayudar a Evágoras en los mismos términos en que posteriormente lo hará Cabrias, es decir, de «mercenario» contratado a título personal, pero con la intención de perjudicar los intereses de su principal «aliado», el Gran Rey, para tratar de reconstruir su imperio marítimo. Jenofonte $(H G, I V, 8,24)$ pone por escrito este episodio presentándolo como una paradoja sin sentido de la política exterior de los atenienses sin llegar a entender (o, si la entendió, no le pareció moralmente correcta) la auténtica intención de Trasíbulo. En cuanto a la actuación de Cabrias a las órdenes de Evágoras, sabemos que ayudó activamente a conquistar el resto de ciudades de la isla para el monarca de Chipre de Salamina (Nepos, Cabrias, II, 2; X, V, 1, 10).

38 Nepos (Cabrias, II) explica que Cabrias lideró la defensa de Egipto por mar con su flota, mientras que Agesilao lo hizo por tierra. Nepos, pues, hace referencia a la expedición griega que participó en la defensa de Egipto en época de Nectanebo durante las campañas persas de los años 362-360, confundiéndola además con la de Farnabazo en 386-384 (Cabrias, III). 
estos contingentes les sumamos las diez naves de Filócrates (X., $H G, I V, 8,24)$ y los tres mil peltastas de Evágoras (siempre y cuando aceptemos que eran mercenarios), concluimos que la cifra exacta de mercenarios griegos reportados por las fuentes es de 23400 soldados de infantería y veinte naves entre 396 y 373. Por tanto, en un período de veinte años fueron reclutados un elevado número de mercenarios para combatir al lado de diferentes Estados en Oriente. Analizándolas de forma aislada, seguramente estas cifras no sean lo suficientemente elevadas como para considerarlas masivas, pero si las comparamos con la participación mercenaria griega en las campañas persas precedentes (incluida la expedición de los Diez Mil) suponen un cambio de modelo en la concepción del mercenario y su contratación, así como un aumento numérico significativo.

\section{CAMBIO DE MODELO}

La campaña egipcio-chipriota resulta de una gran relevancia histórica por varias razones. Al margen de ser el preludio a la «Gran Revuelta de los Sátrapas», constituye el primer caso documentado en que los mercenarios griegos aparecen como una herramienta fundamental en las relaciones internacionales del Mediterráneo Oriental. Su extrema utilidad puede observarse, por ejemplo, en la flexibilidad política que ofrecía el envío de un cuerpo mercenario a los aliados oficiales y, al mismo tiempo, a los enemigos de estos. Es decir, que un Estado obligado a respetar unas reglas de juego (Paz del Rey) que perjudicaban sus intereses podía llevar a cabo de forma encubierta una estrategia que le permitiera vulnerar esas mismas reglas para poder alcanzar así sus aspiraciones políticas reales, que en el caso de Atenas no eran otras que la recuperación de la hegemonía en el Egeo.

En el terreno de la política, los mercenarios contribuyeron al desarrollo de unas necesarias relaciones internacionales, y en el terreno bélico al afianzamiento de nuevas formas de hacer la guerra en Grecia que se ajustaban mejor a la situación política generada por los mencionados pactos internacionales. Así, las póleis griegas se adaptaban a formas menos arcaicas de hacer las guerras, esto es, menos hoplíticas, que habían ido desgastando el sistema sociopolítico de la tradicional pólis $^{39}$. Como se ha podido ver, Cabrias zarpó con un contingente militar para apoyar a Evágoras en su guerra contra el Medo, pero tras la firma de la Paz del Rey, en que Atenas reconocía la soberanía de Artajerjes sobre Chipre, se unió a Ácoris en Egipto, donde trabajó en la fortificación del país (D.S., XV, 29, 1-2). Cabrias, lógicamente, había sido reclutado, oficialmente, a título personal en calidad de mercenario, pero su actuación en Egipto demostró precisamente lo contrario. El general ateniense podía seguir la política que más le interesaba a su ciudad (es decir, la anterior a la Paz del Rey) sin vulnerar los tratados internacionales oficiales a que estaba sujeta. Su propia salida de Chipre mostraría la preocupación de Atenas por convencer al Gran Rey de su voluntad de ser aliados ${ }^{40}$ sin olvidar sus propios

39 En el mundo de la pólis, sobre todo arcaica pero también clásica, la guerra era una cuestión pública de primer orden y, por extensión, la principal manera de participación de un ciudadano de sus obligaciones para con la comunidad. Los mercenarios rompieron esta concepción política en dos aspectos que se entrelazan entre sí, a saber, en primer lugar, luchando por una ciudad que no era la suya y, en segundo lugar, haciendo la lectura inversa, que los ciudadanos permitieran que la defensa de los intereses de su ciudad la llevaran a cabo personas externas a la comunidad. Todo ello favoreció la disolución de la mentalidad aristocrática arcaica en la ciudad, pero, a la larga, también desgastó el sistema mismo de la pólis (Trundle, 2004: 3; Betalli, 1995: 24).

40 Atenas demostraba así tener más control del que se le suponía sobre los mercenarios atenienses, quienes, por su parte, sobre el papel, sólo debían mostrar lealtad por los intereses de sus contratantes. De hecho, el envío de mercenarios fue la fórmula que Lisandro encontró para respaldar la causa de Ciro el Joven de forma oficiosa sin importunar oficialmente ni a los éforos ni a Artajerjes, pues este último era de hecho aliado de Esparta y la potencia lacedemonia, que en aquellos momentos 
intereses al dar cobertura militar a un enemigo de Persia, Egipto. Sin embargo, al parecer la estrategia no consiguió engañar a Farnabazo, que exigió a Atenas la retirada de Cabrias y de todo su contingente mercenario de las tierras egipcias, además del envío de Ifícrates con sus respectivas tropas para que participaran en la campaña en el bando persa. Atenas cedió a las exigencias del sátrapa, lo que en esencia suponía la demostración más evidente de la sumisión ateniense a Persia, puesto que en lugar de «tierra y agua» (es decir, tributos) Atenas contribuyó con soldados. De este modo, Atenas lograba salvar oficialmente parte de su honor al obedecer a Persia obligada por el tratado de paz del 386. La ciudad ática estaba condenada a esconder su política real, tanto en este caso como en el de la construcción de la Segunda Liga de Delos ${ }^{41}$.

Los mercenarios se convirtieron en una pieza fundamental en las relaciones internacionales a principios del siglo IV porque su uso beneficiaba a todas las partes; en primer lugar, podían facilitar las aspiraciones de ciudades griegas como Atenas y Esparta sin vulnerar los tratados internacionales; en segundo lugar, el Gran Rey y los generales persas podían contar con tropas bien entrenadas y bien armadas en muy poco tiempo para sus campañas militares; y por último, suponían un apoyo importante para las potencias que ansiaban sacudirse la soberanía del Gran Rey. Los mercenarios supusieron la evolución del mundo de la guerra o, mejor dicho, la adaptación de ese mundo a un nuevo marco internacional donde los campos de batalla estaban alejados de Grecia y las confrontaciones bélicas iban más allá de disputas territoriales entre Estados vecinos. En esta nueva tesitura de «guerra total», el soldado hoplita (el tradicional ciudadano, propietario y soldado al mismo tiempo) devenía inútil, pues, al final, éste estaba obligado a volver a sus propiedades agrícolas para trabajar la tierra (Cartledge, 1987: 316 ${ }^{42}$. El desarrollo de las relaciones internacionales provocó un cambio en la forma de hacer la guerra que convirtió al mercenario en el soldado ideal. Este proceso ya se había iniciado con la larga Guerra del Peloponeso durante la cual la contratación de mercenarios fue habitual y, así, supuso un punto de inflexión en el modelo de contratación de mercenarios, que desde época arcaica se basaba en los lazos de $\xi \varepsilon v i \alpha$ y $\varphi \imath \lambda i \alpha$ entre grupos aristocráticos (Betalli, 1995: 23-29). De hecho, todas las potencias del Mediterráneo Oriental vieron como el mercenariado podía convertirse en el elemento clave para culminar sus aspiraciones políticas, razón por la cual consideramos que, más incluso que cuestiones endógenas como son la pobreza o la $\varphi \imath \lambda i \alpha^{43}$, ésta fue la causa principal de su imparable crecimiento a partir del siglo IV. Es decir, en nuestra opinión, la evolución en el uso de mercenarios está íntimamente ligada a la intensificación de las relaciones políticas entre los diferentes Estados durante el siglo IV; durante la primera mitad del siglo en relación con la Persia aqueménida, en la segunda a la dominación Macedonia sobre Grecia ${ }^{44}$.

seguía los planes del rey Pausanias, no tenía, a diferencia de la facción de Lisandro, pretensiones hegemónicas en el Egeo ni en Asia Menor. Por lo tanto, al menos oficialmente, no apoyaba a Ciro en su tentativa de asalto a la corona persa (Gómez, 2011).

41 Es decir, respetando la cláusula de la autonomía pero aceptando «donaciones voluntarias» de los aliados. Tras la firma de la Paz del Rey, Atenas buscó inmediatamente alternativas políticas para culminar su proyecto hegemónico (Fornis, 2007: 162), el cual pasaba necesariamente por la formación de una segunda liga áticodélica. En este sentido, el uso de mercancías suponía una alternativa «respetuosa» con los tratados de paz firmados con el persa.

42 El sentido de la expresión «guerra total» en este caso no presupone la implicación de la población civil en los conflictos, sino a la desaparición de la concepción agonística de la guerra, es decir, al enfrentamiento directo entre dos partes ( $\sin$ la participación de terceros) a causa de disputas por la propiedad de la tierra; a la a la estacionalidad y, finalmente, al carácter local de dichas disputas (Lee, 2006: 485).

43 Sin duda estas razones ayudaron a potenciar el fenómeno en el siglo IV (sobre todo el de la pobreza), aunque opinamos que nunca ha sido necesario convertirse en mercenario para sobrevivir a una crisis económica. En cuanto a la relación entre crisis y mercenariado, vid. Miller, 1984.

44 El uso de mercenarios griegos por parte de Darío III está ampliamente documentado en las fuentes (Arr., An., I, 14,4; II, 8, 6; III, 16, 2; Plut, Alex., XVI, 12-15; D.S., XVII, 73, 2). Tras la victoria en Gránico, estos mercenarios griegos 


\section{BIBLIOGRAFÍA}

Alonso, V. (2007): «War, Peace, and International Law in Ancient Greece», K. A. Raaflaub (ed.), War and Peace in the Ancient World. Oxford, Wiley-Blackwell: 206-225.

Antela Bernárdez, B. (2007): «Hegemonía y Panhelenismo: conceptos políticos en tiempos de Filipo y Alejandro». Dialogues d'Histoire Ancienne, 33, 2: 1-21.

Aston, D. A. (1999): «Dynasty 26, Dynasty 30, or Dynasty 27? In Search of the Funerary Archeology of the Persian Period», A. Leahy y J. Tait (eds.), Studies on Ancient Egypt in Honour of H.S. Smith. London, Egypt Exploration Society: 17-22.

Betalli, M. (1995): I mercenari nel mondo greco. Dalle origini alla fine del V sec. a.C. Pisa, Edizioni ETS.

Bostworth, A.B. (1989), 'Autonomia: the Use and Abuse of Political Terminology', Studi Italiani di Filologia Classica 85, 122-151.

Briant, P. (1988): «Ethno-classe dominante et populations soumises dans l'Empire Achemenide: le cas de l'Egypte», A. Kuhrt y H. Sancisi-Weerdenburg (eds.), Method and Theory. Achaemenid History, 3: $137-173$.

Briant, P. (1996): Histoire de l'empire perse. De Cyrus à Alexandre. Paris, Libraire Arthème Fayard.

Briant, P. (2003): «New Trends in Achaemenid History». The Ancient History Bulletin, 117, 1-2: $33-$ 47.

Briant, P. (2006): La història de l'Imperi Persa (550-330), J. Curtis y N. Tallis (eds.), L'Imperi Oblidat. El món de l'antiga Pèrsia. Barcelona, Fundació la "Caixa".

Cartledge, P. (1987): Agesilaos and the crisis of Sparta. Baltimore, The Johns Hopkins University Press.

Cawkwell, G. L. (1976): «The Imperialism of Thrasybulus». The Classical Quarterly, New Series, 26, 2: $270-277$.

Cawkwell, G. L. (1981): «The King's Peace». The Classical Quarterly, New Series, 31, 1: 69-83.

Fornis, C. (2007): «La paz enviada por el Rey, 387/368 a.C.». Dike. Rivista di storia del diritto greco ed ellenistico, 10: 155-183.

Fornis, C. (2008): Grecia Exhausta. Ensayo sobre la guerra de Corinto, Göttingen.

García Sánchez, M. (2005): «La figura del sucesor del Gran Rey en la Persia aqueménida». Gerión Anejos, IX: 223-239.

Garlan, Y. (1972): La Guerre dans l'Antiquité. Paris, F. Nathan.

Gómez Castro, D. (2008): Los mercenarios griegos en el Imperio Aqueménida en el siglo IV a.C.: la revuelta egipcio-chipriota y los precedentes a la Gran Revuelta de los Sátrapas (385-373). Barcelona, Universitat Autònoma de Barcelona (tesis inédita).

Gómez Castro, D. (2010), 'El mercenario en el mundo griego a la luz de los estudios contemporáneos: reflexión teórica y nuevas tesis, Habis 41, 95-116.

Gómez Castro, D. (2011), 'Trabajar para el enemigo: Jenofonte a la luz de la investigación reciente', en Cortés, J.M, Muñiz, E., Gordillo, R. (eds), Actas de la $V^{a}$ Reunión del Historiadores del mundo griego antiguo, Sevilla, 2011 (en prensa).

Hansen, M. H. (1995): «The "autonomous" city-state. Ancient fact or modern fiction?», M. H. Hansen y K. Raaflaub (eds.), Studies in the Ancient Greek Polis. Papers from the Copenhagen Polis Centre, 2. Stuttgart, Historia Einzewlschriften, 95: 21-43.

Karavites, P. (1984): «The political use of ELEUTHERIA and AUTONOMIA in the fourth Century among the Greek city states». Revue Internationale des Droits de l'Antiquité, 31: 167-191.

Landucci, F. (1994): «I mercenary nella politica ateniense dell'etá di Alessandro». Ancient Society, 25: 33-61.

Lee, J. W. I. (2006): «Warfare in the Classical Age», K. H. Kinzl (ed.), A Companion to the Classical Greek World. Oxford, Blackwell Companions to the Ancient World, Blackwell-Malden: 480-508.

fueron condenados a trabajos forzados en Macedonia por su «desesperado coraje» en su lucha contra los macedonios (Landucci, 1994: 35; Plut., Alex., XVI, 12-15) y, a juzgar por la reacción filopersa de Atenas antes de la batalla de Issos (Arr., An., II, 15, 2; Landucci, 1994: 37), ha habido autores que han considerado que la mayoría de los mercenarios griegos que se enfrentaron a Alejandro lo hicieron para intentar sacudir el yugo macedonio de la Hélade (Trundle, 2004: 71). 
Mallet, D. M. (1922): Les rapports des grecs avec l'Egypte (de la conquête de Cambise, 525, a celle d'Alexandre, 334. Caire, Institut Française d'Archéologie Orientale. (datos de http://openlibrary. org)

Marinovic, L. P. (1997): «La vocation politique des mercenaires». Cahiers des études anciennes, XXXIIII: 119-121.

Miller, H. (1984): «The Practical and Economic Background to the Greek Mercenary Explosion». Greece \& Rome, 31, 2: 153-160. (datos finales de http://www.jstor.org/stable/642581)

Moritani, K. (1988), 'KOINE EIRENE: Control, Peace, and 'Autonomia' in Fourth-Century Greece', Yuge, T., Doi, M. (eds), Forms of Control and Subordination in Antiquity, Leiden, 573-577.

Olmstead, A.T. (1948), History of the Persian Empire, Chicago.

Orsi, D. P. (2004): «Sparta e la Persia. La guerra in Asia, 400-394 a.C.». Incidenza dell’antico. Dialoghi di storia greca, 2: 41-58.

Pascual González, J. (1986), 'El surgimiento de una facción democrática tebana', Faventia 8, 69-83.

Pascual González, J. (1991): «Las facciones políticas tebanas en el período de formación de la hegemonía (379-371 a.C.). I.- La conspiración democrática del 379», Polis. Revista de ideas y formas políticas de la Antigüedad Clásica, 3: 121-135.

Pascual González, J. (1995): «Corinto y las causas de la Guerra de Corinto». Polis. Revista de ideas y formas políticas de la Antigüedad Clásica, 7: 187-218.

Petit, T. (1991): «Presence et influence perses à Chypre», H. Sancisi-Weerdenburg and A. Kuhrt (eds.), Achaemenid History, VI. Asia Minor and Egypt: Old cultures in a new Empire. Leiden, Nederlands Instituut voor het Nabije Oosten: 161-178. (datos de http://www.nino-leiden.nl/ Proceedings of the Groningen 1988 Achaemenid History Workshop)

Plácido Suárez, D. (2001-2002): «La "autonomía" de las ciudades griegas». Veleia, 18-19: 201-209.

Ryder, T. T. B. (1965): Koine eirene. Oxford, Clarendon Press (datos de http://journals.cambridge. org).

Sato, N. (2006): «Athens, Persia, Clazomenae, Erythrae: an Analysis of International Relationships in Asia Minor at the Beginning of the fourth Century BCE». Bulletin of the Institute of Classical Studies, 49: 23-37.

Schmidt, K. (1999): «The Peace of Antalcidas and the Idea of the "koine eirene". A Panhellenic Peace Movement». Revue internationale des droits de l'Antiquité, 46: 81-96.

Seager, R. (1974), 'The King's Peace and the Balance of Power in Greece, 386-362 B.C.', Athenaeum 52, 36-63.

Seibt, F. (1977): Griechische Söldner im Achaimenidenreich. Habelt, Bonn.

Shrimpton, G. (1991): «Persian Strategy Against Egypt and the date for the Battle of Citium», Phoenix, 45: $1-20$.

Stylianou, P. J. (1998): A Historical Commentary on Diodorus Siculus. Book 15. Oxford, Clarendon.

Tourraix, A. (1999): «Les mercenaires grecs au service des Achéménides», P. Brun (coord.), Questions d'Histoire. Guerres et Sociétés dans les mondes grecs (490-322). Paris. Éditions du temps: 201216.

Trundle, M. (2004): Greek Mercenaries, from the Late Archaic Period to Alexander. London/New York, Routledge.

Van Der Speck, R. J. (1998): «The Chronology of the wars of Artaxerxes II in the Babilonian Astronomical Diaries», Achaemenid History, 11: 239-256.

Recibido: 09/02/2009

Aceptado: 12/05/2009 